\title{
Clasificación de los bolsillos de retracción timpánica del Hospital Barros Luco-Trudeau
}

\section{Classification of Barros Luco Hospital's tympanic pockets retraction}

\author{
Ramón Readi S', Úrsula Zelada B¹, Esteban Ortúzar G'.
}

\begin{abstract}
RESUMEN
Introducción: Los bolsillos de retracción póstero-superiores de la pars tensa (BR) pueden progresar con erosión osicular y colesteatoma. Se han descrito una serie de mecanismos fisiopatológicos que explican la mayor ocurrencia de retracciones en esta zona. En el Hospital Barros Luco se utiliza una clasificación, variación a la de Sadé, que representaría mejor la progresión de la enfermedad, junto a una propuesta de tratamiento.

objetivo: Aplicar nuestra clasificación de los BR y describir clínica y audiológicamente cada grado junto a los tratamientos indicados. Clasificar y describir cada grado según: PTP óseo y aéreo, primera cirugía, progresión de la enfermedad, otorrea, hipoacusia, OME y erosión osicular.

Material y método: Estudio retrospectivo y descriptivo de 83 pacientes y 92 oídos presentados al Departamento de Oído del Servicio de Otorrinolaringología del Hospital Barros Luco más la revisión de su ficha clínica y del primer protocolo operatorio entre Ios años 2005 a 2009. Con seguimiento mínimo de 12 meses. Variables: Edad, sexo, PTP óseo y aéreo en 4 frecuencias, primer procedimiento quirúrgico, progresión de la enfermedad, otorrea, hipoacusia, OME y erosión osicular.

Resultados: La hipoacusia y los PTP aéreos y óseos no presentaron diferencias entre los grados. Hubo una gradiente de disminución de OME desde el grado I al V. La presencia de otorrea fue aumentando progresivamente hasta el grado IV, disminuyendo discretamente en el V. Hubo un aumento de la presencia de erosión de la rama larga del yunque desde 0 en el grado I hasta 64,3\% en el grado IV. En los tratamientos, la timpanectomía más algún tipo de tubo de ventilación con o sin adenoidectomía, representó el 43,5\% en el grado III. En el grado IV el procedimiento más frecuentemente efectuado fue la aticotomía con $40 \%$.

Conclusiones: Esta clasificación reflejaría grados de gravedad de los $B R$ en aspectos como: otorrea, erosión osicular y tratamiento propuesto. La presencia de hipoacusia y el PTP aéreo no reflejan el grado de progresión de la enfermedad. Se propone una modificación a nuestra clasificación.
\end{abstract}

Palabras clave: Bolsillos de retracción, atelectasia timpánica.

${ }^{1}$ Médico. Servicio de Otorrinolaringología. Hospital Ramón Barros Luco-Trudeau. 


\section{ABSTRACT}

Introduction: Pockets of posterior-superior retraction of the pars tensa (BR) can progress with ossicular erosion and cholesteatoma. Has been described a series of pathophysiological mechanisms that explain the higher occurrence of retraction in this area.In the Barros Luco Hospital we use a classification, a modification of Sadé, that would represent best the progression of the disease together to a treatment proposed.

Aim: Applying our classification of BR and describe clinical and audiologically each grade with the indicated treatments. Classify and describe each grade by: PTP bone and air, first surgery, disease progression, otorrhea, hearing loss, ossicular erosion and OME

Material and method: Retrospective and descriptive analysis of 83 patients and 92 earssubmitted to the Department of Ear of Otolaryngology Service's Hospital Barros Luco, plus review of the clinical record and the first surgical protocol between the years 2005 to 2009. Minimum follow-up of 12 months. Variables: age, sex, and air bone PTP in 4 frequencies, first surgical procedure, disease progression, otorrhea, hearing loss, ossicular erosion and OME.

Conclusion: This classification would reflect degrees of severity of $B R$ in aspects such as otorrhea, ossicular erosion and proposed treatment. The presence of hearing loss and air PTP does not reflect the degree of disease progression. An amendment to our classification is proposed.

Key words: Pockets retractions, tympanic atelectasis.

\section{INTRODUCCIÓN}

Los bolsillos de retracción póstero-superiores de la pars tensa o retracción de la pars tensa hacia o dentro del ático 0 , simplemente, bolsillos de retracción (BR), se definen ${ }^{1}$ como una depresión de parte del tímpano, con una amplia abertura, situada principalmente en el cuadrante póstero-superior (PS) del tímpano. No son infrecuentes y pueden progresar con erosión osicular y colesteatoma. Representan un dilema otológico: no existe consenso de cuál es el tratamiento ideal a pesar de la incrementada comprensión de la enfermedad².

Como mecanismo fisiopatológico de desarrollo de un BR, se le ha atribuido un rol a la disfunción tubaria y al debilitamiento de la membrana timpánica debido a otitis media con efusión (OME). $\mathrm{Ars}^{3}$ mostró que el $60 \%$ de las retracciones se ubican en la pars tensa ( $36 \%$ en el cuadrante PS y $16 \%$ en la mitad inferior), mientras que el $40 \%$ restante están en la pars fláccida. La razón de por qué la pars tensa PS está en mayor riesgo, puede ser explicada por un estudio histopatológico de huesos temporales de niños menores de tres años 6 meses. Se demostró que en los niños mayores de tres años con enfermedad inflamatoria del oído, había persistencia de mesénquima en esta porción de la membrana timpánica, siendo que normalmente se reabsorbe a los 2 meses de vida. Como consecuencia, existe una pobre maduración de elastina en esta región, generando una membrana timpánica débil, una vez que se reabsorbe este mesénquima ${ }^{3}$.

La pars fláccida es inherentemente débil, comparado al resto de la membrana timpánica debido a que carece de capa fibrosa, aumentando el riesgo de retracción y colesteatoma atical ${ }^{3}$.

La pars tensa tiene tanto fibras colágenas circulares y radiadas que están bien organizadas y relativamente densas. La porción posterior de la pars tensa tiene una lámina propia más delgada que la anterior y también tiene un riego sanguíneo aumentado. La lámina propia de la porción PS tiene fibras colágenas más delgadas y escasas, dispuestas de forma circular ${ }^{4}$.

Su riego sanguíneo más aumentado la hace más propensa a daño, debido a procesos inflamatorios. Éstos alteran el soporte estructural de colágeno, volviendo al tímpano débil y más propenso a retracciones ${ }^{4}$. 
A comienzos de la década de los 90, el Dr. Luis Dentone ${ }^{5}$, inspirado en Sadée-8, propuso una clasificación más práctica que estuviera ligada a una conducta a seguir frente a cada BR. Es más esquemática, simple y representaría una progresión de la enfermedad y, además, tiene ligada una conducta a seguir. Es de uso habitual en nuestro centro, se enseña en el curso de posgrado y no ha sido publicada:

- Grado I: Bolsillo PS leve

- Grado II: Bolsillo PS que contacta la I-E

- Grado III: Bolsillo PS de fondo no visible

- Grado IV: Bolsillo PS que retiene piel

- Grado V: Otopatía adhesiva

Bolsillo evolutivo: Es cualquier bolsillo que progresa en grado o que presenta deterioro auditivo.

Como se muestra en la Tabla 1, Ios III y IV son descritos como "bolsillos peligrosos", Ios cuales junto a los "bolsillos evolutivos", implican una conducta activa.

\section{OBJETIVO}

Aplicar nuestra clasificación de los BR y al describir clínica y audiológicamente cada grado definir conducta 0 tratamiento.

Clasificar y describir cada grado según PTP óseo y aéreo, primer procedimiento quirúrgico realizado, presencia de progresión de la enfermedad postratamiento, otorrea, hipoacusia, OME y erosión del yunque.

\section{MATERIAL Y MÉTODO}

Es un estudio retrospectivo y descriptivo de los casos presentados al Departamento de Oído del Servicio de Otorrinolaringología del Hospital Barros Luco más la revisión de la ficha clínica y del primer protocolo operatorio entre los años 2005 a 2009. Fueron 83 pacientes y 92 oídos con un seguimiento mínimo de 12 meses. Las variables estudiadas fueron: edad, sexo, PTP óseo y aéreo en 4 frecuencias, primer procedimiento quirúrgico, presencia de progresión de la enfermedad, otorrea, hipoacusia, otitis media con efusión (OME) y erosión osicular.

Para el análisis de los resultados se aplicó un $\alpha=0,05$, la prueba de Fisher para la distribución de proporciones, la prueba T para análisis de variables continuas. ANOVA, Wilcoxon y Mann-Withney, para la comparación de promedios tonales puros pre y postratamiento. Se utilizó el programa estadístico SPSS 17. Se excluyeron los datos incompletos, la otitis media crónica perforada y la colesteatomatosa.

\section{RESULTADOS}

Se estudiaron 83 pacientes con un total de 92 oídos, con un promedio de edad 26,8 años (3-79); DE 21,1 años. El promedio de meses de seguimiento fue 45,7 meses (12-144); DE 29,2. Sin diferencias en sexo y lado afectado.

En la Figura 1, podemos ver que al aplicar la clasificación del Hospital Barros Luco, el BR más

Tabla 1. Esquema de tratamiento de los bolsillos de retracción (L Dentone, 1990) ${ }^{5}$

\begin{tabular}{|l|l|}
\hline \multicolumn{1}{|c|}{ Grado de retracción } & \multicolumn{1}{c|}{ Tratamiento propuesto } \\
\hline Grado I estable & Observación \\
\hline Grado I evolutivo & Tubo en cuadrante anterosuperior \\
\hline Grado II estable & Observación \\
\hline Grado II evolutivo & Tubo y/o timpanectomía \\
\hline Grado III & Timpanectomía ${ }^{9}$ y tubo \\
\hline Grado IV & Timpanectomía y tubo. Aticotomía + timpanoplastía c/s tubo \\
\hline
\end{tabular}


frecuente fue el grado II con $39,8 \%$, el grado III con $27,7 \%$, el grado IV con $18,1 \%$. El grado I y el IV con $7,2 \%$ cada uno.

Del grupo total solo 4 pacientes se presentaron como un bolsillo evolutivo. Dos pacientes en grado II por deterioro auditivo y uno con grado IV y otro con grado $\mathrm{V}$ por progresión del bolsillo.

En la Figura 2, se analiza la presencia de hipoacusia, OME y otorrea. La hipoacusia no presentó diferencias entre los grados ( $p$ de Fisher; $p=0,21$ ). Hubo una gradiente de disminución estadísticamente significativa de la presencia de OME desde el grado I al V ( $p$ de Fisher; $p<0,01$ ). A la inversa, la otorrea fue aumentando progresivamente de manera estadísticamente significativa hasta el grado IV, volviendo a disminuir discretamente al V ( $p$ de Fisher; $p<0,01$ ).

Los PTP aéreos y óseos a través de los grados de la clasificación no presentaron diferencias estadísticamente significativas. Tampoco hubo diferencias estadísticamente significativas al controlar por OME y erosión del yunque (ANOVA; $p=0,74$ ).

Como muestra la Figura 3, se encontró un aumento estadísticamente significativo de la presencia de erosión de la rama larga del yunque desde 0 en el grado I hasta $64,3 \%$ en el grado IV. El grado $V$ se comportó como una entidad diferente con $33,3 \%$ ( $p$ de Fisher; $p=0,03$ ).

Figura 3. Revela la distribución porcentual de erosión de la rama larga del yunque según clasificacion de los BR del Hospital Barros Luco ( $p$ de Fisher; $p=0,03) n=92$.

En la Figura 4 se puede observar que hubo un gradiente de disminución de la presencia de pacientes sometidos a observación, desde el grado I con $66,7 \%$ al grado IV con $13,3 \%$. Nuevamente el grado $\mathrm{V}$ se comportó como una entidad diferente con $50 \%$ ( $p$ de Fisher; $p<0,01$ ).

En la Figura 5 se observan los diferentes esquemas de tratamiento que se realizaron frente a un bolsillo grado III. La observación representó el $34,8 \%$ de los casos. La timpanectomía ${ }^{9}$ más el tubo en T el 26,1\%. La timpanectomía más algún tipo de tubo de ventilación con o sin adenoidectomía, representó el 43,5\%.

En la Figura 6 vemos que, en el caso del grado IV, el procedimiento más frecuentemente efectuado fue la aticotomía con $40 \%$.

Al analizar la presencia de progresión de la enfermedad posterior al primer tratamiento, la mitad

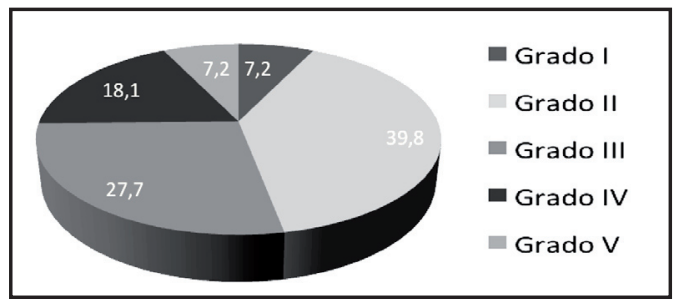

Figura 1. Porcentajes de distribución según la clasificación de los BR del Hospital Barros Luco $(n=92)$.

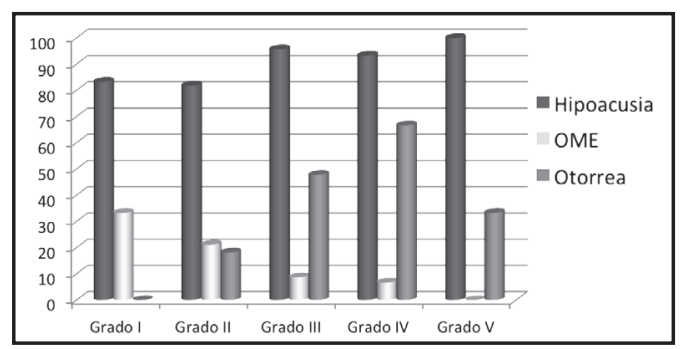

Figura 2. Distribución de manifestaciones clínicas según clasificación de los BR del Hospital Barros Luco $(n=92)$.

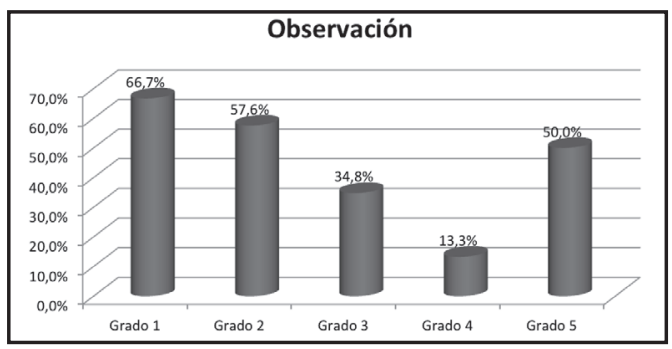

Figura 3. Distribución porcentual de pacientes bajo observación según clasificación del Hospital Barros Luco $(n=92)$.

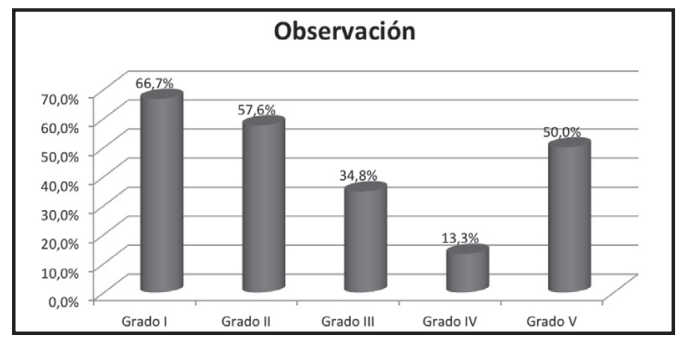

Figura 4. Distribución porcentual de pacientes sometidos a observación según la clasificación del Hospital Barros Luco ( $p$ de Fisher; $p<0,01) n=92$.

de los pacientes con grado I progresaron, presentando porcentajes menores en los restantes con $15,2 \%$ en el grado II, $17,4 \%$ en el III, $6,7 \%$ en el IV y $16,7 \%$ en el V. La presencia de perforación residual 

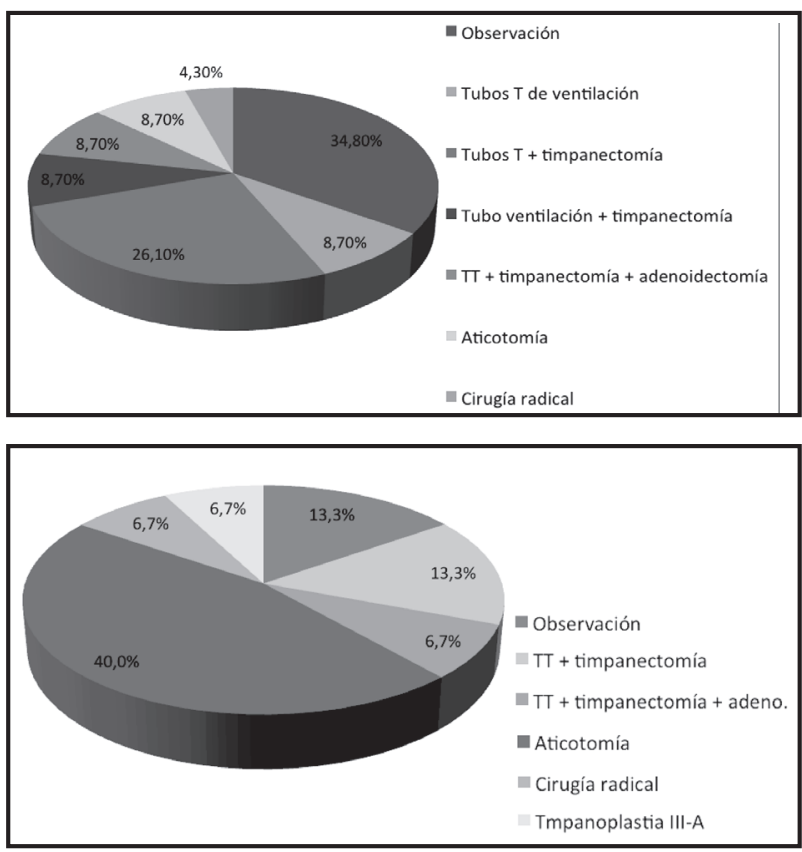

Figura 5. Distribución porcentual de los tipos de tratamientos en el grado III de la clasificación del Hospital Barros Luco.

Figura 6. Distribución porcentual de los tipos de tratamientos en el grado IV de la clasificación del Hospital Barros Luco. postratamiento fue muy baja alcanzando un máximo en el grado $V$ con $16,7 \%$ ( $p$ de Fisher; $p=0,4$ ).

No se encontraron diferencias estadísticamente significativas al comparar los PTP aéreos pre y postratamientos (prueba $T$ y de Wilcoxon; $p$ $>0,05$ ), tampoco al analizar en el subgrupo de timpanectomía ${ }^{9}$ más tubo en T.

\section{DISCUSIÓN}

La clasificación utilizada en nuestro servicio reflejaría grados de gravedad de los BR en aspectos como: otorrea, erosión osicular y tratamiento propuesto.

Sin embargo, la presencia de hipoacusia y el PTP aéreo son elementos poco fiables para guiar el grado de progresión de la enfermedad, ya que no encontramos diferencias estadísticamente significativas entre los grados.

El grado V, es decir, la otitis fibroadhesiva, se comportó como una entidad distinta a los grados que la preceden al analizar su comportamiento por presencia de OME, otorrea, necrosis de la rama larga del yunque y presencia de perforación residual postratamiento. En este grado, es en donde más frecuentemente se produjo esta última complicación. Además, hubo un gradiente progresivo de disminución de los pacientes que fueron observados al avanzar a través de los grados, sin embargo, en el grado 5 esta postura volvió a subir con el $50 \%$.

Solo 4 pacientes se presentaron con bolsillo evolutivo, explicado quizás porque la escala temporal de la evolución natural de esta enfermedad es de muchos años como para ser presenciada por un observador.

\section{CONCLUSIONES}

Desde el punto de vista de nuestra clasificación, independientemente de las características de la enfermedad atelectásica del resto del tímpano de la pars tensa, la conducta fue definida por las características del BR.

Hemos clasificado y descrito cada grado según PTP óseo y aéreo, primer procedimiento quirúrgico realizado, presencia de progresión de la enfermedad postratamiento, otorrea, hipoacusia, OME y erosión del yunque.

No encontramos una uniformidad de tratamientos propuestos de acuerdo a cada grado. Esto es concordante con reportes internacionales que indican que no existe aún un consenso sobre el momento, las indicaciones y las opciones de tratamientos propuestos ${ }^{10}$.

Resulta fundamental tener en consideración la progresiva mayor presencia de erosión osicular a medida que empeora el grado, porque implica mayor dificultad quirúrgica y, que por lo tanto, su resolución debe estar en manos de cirujanos más entrenados. 


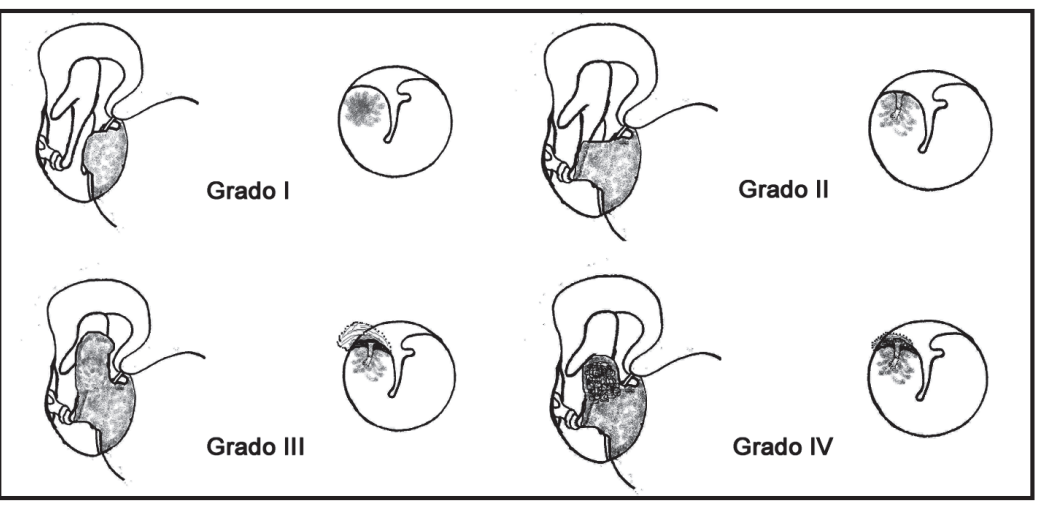

Figura 7. Propuesta de modificación de la clasificación de los BR del Hospital Barros Luco.

Por todo lo anterior, se puede plantear incluir dentro del ejercicio de la clasificación, la identificación y descripción de erosión de la rama larga del yunque.

Además, proponer la omisión del grado V (otitis fibroadhesiva) por corresponder a una entidad distinta a los BR.

\section{PROPOSICIÓN}

En la Figura 7, podemos ver una propuesta de modificación a esta clasificación, que puede ser como se muestra a continuación:

Bolsillo No Peligroso:

- Grado I: Bolsillo PS leve.

- Grado II: Bolsillo PS que contacta la I-E, con 0 sin erosión del yunque.

Bolsillo peligroso:

- Grado III: Bolsillo PS de fondo no visible.

- Grado IV: Bolsillo PS que retiene piel.

- Bolsillo evolutivo: que progresa en grado o presenta deterioro auditivo.

\section{BIBLIOGRAFÍA}

1. Rondón H. Cap. 5.2.3A: Atelectasis and retraction pockets of the tympanic membrane. Goycoolea M. Atlas of Otologic Surgery and Magic Otology Volumen 1, 515-20. JP Medical Ltd, 2012.
2. Ramakrishnan $Y, A$ Kotecha $A$, Bowdler $D$ A. A review of retraction pockets: past, present and future management. The Journal of Laryngology \& Otology 2007; 121: 521-5.

3. Steinbach E, Pusalkar A, Heumann H. Cholesteatoma -pathology and treatment. Adv Otorhinolaryngol 1988; 39: 94-106.

4. Danner C. Middle Ear Atelectasis: What Causes It and How Is It Corrected? Otolaryngol Clin N Am 2006; (39): 1211-9.

5. Zelada U, Dentone L, Contador AM, Dentone P, Moreno M. Evaluación del tratamiento quirúrgico de los bolsillos de retracción timpánica. CEPE Hospital Barros Luco. Septiembre de 2006.

6. Sadé J, Avraham S, Brown M. Atelectasis, retraction pockets and cholesteatoma. Acta Otolaryngol 1981; 92: 501-12.

7. Borgstein J, Gerritsma TV, Wieringa MH, Bruce IA. The Erasmus atelectasis classification: proposal of a new classification for atelectasis of the middle ear in children. Laryngoscope 2007; 117: 1255-9.

8. Sadé J, Berco E. Atelectasis and secretory otitis media. Ann Otol Rhinol Laryngol 1976; 85(2) (suppl 25, pt 2): 66-72.

9. Bonilla G. Timpanectomía en retracciones timpánicas. Rev Otorrinolaringol Cir Cabeza Cuello 1998; 58(3): 135-9.

10. Nankivell P, Pothier D. Surgery for tympanic membrane retraction pockets. The Cochrane Library 7 Jul 2010.

\section{Dirección: Ramón Readi Sakurada \\ Servicio de Otorrinolaringología. Hospital Ramón Barros Luco-Trudeau Gran Avenida José Miguel Carrera 3204. San Miguel, Santiago \\ Email: areadis@sochiorl.cl}

